\title{
25
}

\section{ELLIPTIC CURVE CRYPTOSYSTEMS ON SMART CARDS}

Elsayed Mohammed, A. Emarah and Kh. El-Shennawy

Arab Academy for Science and Technology, Air Defense College, EGYPT

Abstract: Elliptic Curve Cryptography (ECC) offers small key size and high security. Secure applications in smart cards present implementation challenges particular to the platform's memory, bandwidth, and computation constraints. ECC's unique properties make it especially well suited to smart card applications. ECC systems provide the highest strength per bit of any cryptosystem known today. This paper presents a new protocol for smart card implementation of elliptic curves explaining how ECC can not only significantly reduce the cost, but also accelerate the deployment of smart cards in new applications.

Key words: $\quad$ smart card, elliptic

\section{INTRODUCTION}

Smart cards are proposed for use in a wide variety of applications such as electronic commerce, identification, and health care. For many of these proposed applications, cryptographic services offered by digital signatures would be required. Smart cards also need to be inexpensive. Recently, public key cryptography (PKC) has gained increasing attention from both companies and end users to add security to a wide variety of applications. Today, three types of public key systems, classified according to the mathematical problem on which they are based, are generally considered both secure and efficient [1] [2] [3]. The systems are:

- Integer factorization systems (of which RSA is the best known example)

- Discrete logarithm systems (such as the U.S. Government's DSA) 
- Elliptic curve discrete logarithm systems (also known as elliptic curve cryptosystems).

\subsection{Smart Card Implementation Constraints and ECC}

Smart cards present a combination of implementation constraints that other platforms do not: constrained memory and limited computing power are two of them. The majority of the smart cards on the market today have between 128 and 1024 bytes of RAM, 1 and 16 kilobytes of EEPROM, and 6 and 16 kilobytes of ROM with the traditional 8-bit CPU typically clocked at about 3.57 megahertz. Any addition to memory or processing capacity increases the cost of each card because both are extremely cost sensitive. The following points summarize the key benefits of using ECC on smart cards.

- Less EEPROM and shorter transmission times - The strength (difficulty) of the ECDLP algorithm means that strong security is achievable with proportionately smaller key and certificate sizes. The smaller key size in turn means that less EEPROM is required to store keys and certificates and that less data needs to be passed between the card and the application so that transmission times are shorter.

- Scalability - As smart card applications require stronger and stronger security (with longer keys), ECC can continue to provide the security with proportionately fewer additional system resources. This means that with ECC, smart cards are capable of providing higher levels of security without increasing their cost.

- No coprocessor - The crypto coprocessors used in some cryptosystems not only take up precious space - they add about 20 to 30 percent to the cost of the chip, and about three to five dollars to the cost of each card. With ECC, the algorithm can be implemented in available ROM, so no additional hardware is required to perform strong, fast authentication.

\subsection{Security Requirements}

We describe the security and efficiency features desirable in modern communication systems and devices.

- Mutual authentication - One of the most important aspects of smart card usage is mutual authentication. The system should be designed to avoid using a fake smart card that deceives the terminal to steal money or gain 
illegal access to a protected resource. The fraud can also come from the other side by using a hostile terminal that transfers the deducted money, in a financial transaction, to a thief not to the real seller.

- Non-repudiation of service - For a perfectly designed smart card system, it should not be possible for users to deny the charges occurred by the use of service. This feature can easily be implemented in security protocols by the use of digital signatures. Elliptic curve digital signatures are the most efficient among public key signature schemes.

- Confidentiality - The smart card-to-terminal communication process is well secured against eavesdropping. If the terminal is connected to a bank or a host (in online transactions), this conversation should also be secured against passive and active attacks. The participants of such conversations must agree on a key that can be used to encrypt messages. Key agreement is usually the last part of the authentication process between smart cards and terminals. Session keys must differ for each transaction to enhance security.

- Anonymity of user - In a communication setting, some users may not want to expose their identities and/or their locations to third parties [4]. In traditional phone systems, once the call is set up, the identities of the users are automatically exposed to the servers. In smart card systems, an authentication protocol can be designed in a way that every user is assigned a temporary identity when they get their certificate from the Certificate Authority (CA). Then, the assigned identities can be used in that domain. The proposed protocol in this paper hides the identity of the cardholder from the server.

- Physical requirements - Limited size chips are used to store the keys and run the algorithms. This requires that the complexity of the cryptographic operations should be minimal. Factors like hardware complexity and computational delay play important roles in the design of public key protocols for smart cards. Storage requirement is the most important factor, since we often need to store the public keys, certificates, and temporary data used during the execution of the protocol. Storage may not be a severe factor for the server side but it is a highly important for the smart card side.

\section{ELLIPTIC CURVE CRYPTOGRAPHY}

The protocol described in this paper depends on the security of the elliptic curve primitives, e.g., key generation, signature generation, and signature 
verification. These operations utilize the arithmetic of points which are elements of the set of solutions of an elliptic curve equation defined over a finite field. The security of the protocol depends on the intractability of the elliptic curve analogue of the discrete logarithm problem, which is a well known and extensively studied computationally hard problem. Basic elliptic curve arithmetic operations are described in the appendix.

\subsection{Basic Definitions and Notation}

We first define the terms and then provide a general overview of the notations used in this paper:

- Secret Key Algorithm: The secret key encryption and decryption operations using the key $K$ acting on the plaintext $M$ and the ciphertext $C$ are denoted as: $C=E(K, M)$ and $M=D(K, C)$, respectively.

- Message Digest Function: We will denote the message digest of a message $M$ by $H(M)$. The signature functions take $H(M)$ as an input for efficiency reasons. The hash of the concatenation of two messages $M_{1}$ and $M_{2}$ is denoted as $H\left(M_{1}, M_{2}\right)$.

\subsection{Elliptic Curve Digital Signature Algorithm}

First, an elliptic curve $E$ defined over $G F(p)$ or $G F\left(2^{k}\right)$ with large group of order $n$ and a point $P$ of large order is selected and made public to all users. Then, each party uses the following key generation primitive to generate the individual public and private key pairs. Furthermore, for each transaction the signature and verification primitives are used. Elliptic Curve Digital Signature Algorithm (ECDSA) [6] is outlined below.

\section{- ECDSA Key Generation:}

The user A follows these steps:

1. Select a random integer $d \in[2, n-2]$.

2. Compute $Q=d^{*} P$.

3. The public key of the user $A$ is $(E, P, n, Q)$ and the private key is $d$.

\section{- ECDSA Signature Generation:}

The user $A$ signs the message $\mathrm{m}$ using the following steps:

1. Select a random integer $k \in[2, n-2]$.

2. Compute $k * P=\left(x_{1}, y_{1}\right)$ and $r=x_{1} \bmod n$. If $x_{1} \in \mathrm{GF}\left(2^{k}\right)$, it is assumed that $x_{1}$ is represented as a binary number. 
If $r=0$ then go to Step 1 .

3. Compute $k^{-1} \bmod n$.

4. Compute $s=k^{-1}(H(m)+d . r) \bmod n$.

Here $H$ is the secure hash algorithm SHA.

If $s=0$ go to Step 1 .

5. The signature for the message $m$ is the pair of integers $(r, s)$.

\section{- ECDSA Signature Verification:}

The user $B$ verifies $A$ 's signature $(r, s)$ on the message $m$ by applying the following steps:

1. Compute $c=s^{-1} \bmod n$ and $H(m)$.

2. Compute $u_{1}=H(m) . c \bmod n$ and $u_{2}=r . c \bmod n$.

3. Compute $u_{1} * P+u_{2} * Q=\left(x_{0}, y_{0}\right)$ and $v=x_{0} \bmod n$.

4. Accept the signature if $v=r$.

\section{PROPOSED PROTOCOL FOR SMART CARDS}

It is assumed that there is a certificate authority (CA) which creates and distributes certificates to the users and servers on their request. These certificates contain a temporary identity assigned by the $\mathrm{CA}$ for the requesting party, the public key of the requesting party, and the expiration date of the certificate. The concatenated binary string is then signed by the CA's private key to obtain the certificate for the requesting party. By using a certificate the identity of a particular party is bound to its public key. The acquisition of the certificate is performed when the users and servers first subscribe to the service. The certificates are updated at regular intervals, for example, in the beginning of each month after paying the monthly charge. Note that in this discussion the term 'Server' is equivalent to 'Terminal' and the term 'User' is equivalent to 'smart card'.

\subsection{Terminal and Card Initializations}

In order to receive a certificate, the terminal sends its public key $Q_{s}$ together with its user identity through a secure and authenticated channel to the CA. The CA uses its private key to sign the hashed value of the concatenation of the public key, the temporary identity $I_{s}$, and the certification expiration date $t_{s}$. The CA then sends the signed message through the secure and authenticated channel to the terminal as shown in Figure 1. 
TERMINAL OR SERVER

- Choose $d_{s} \in[2, n-2]$

- $Q_{s}=d_{s} * P$

- Send

- Receive

- $e_{s}=H\left(Q_{s} \cdot x, I_{s}, t_{s}\right)$

- Store $Q_{s}, Q_{c a}, I_{s}\left(r_{s}, s_{s}\right), e_{s}, t_{s}$ on the terminal

Establishing a secure channel from the certification authority to the terminal is a common and accepted assumption in almost all authentication protocols. The signed message is the certificate of the terminal which is used in future authentication and key generation processes. By repeating the very same process the smart card acquires its certificate as shown in Figure 2.

CARD

- Choose $d_{u} \in[2, n-2]$

- $Q_{u}=d_{u} * P$

- Send
CERTIFICATION AUTHORITY

- Choose $k_{u} \in[2, n-2]$

- $R_{u}=k_{u} * P$

- Receive

- Choose unique $I_{u}$

- $r_{u}=R_{u} \cdot x$

- $s_{u}=k_{u}^{-1}\left(H\left(Q_{u} \cdot x, I_{u}, t_{u}\right)+d_{c a} \cdot r_{u}\right)$

- Receive

- $e_{u}=H\left(Q_{u} \cdot x, I_{u}, t_{u}\right)$

$Q_{c a}, I_{s},\left(r_{u}, s_{u}\right), t_{u} \quad \bullet$ Send

- Store $Q_{u}, Q_{c a}, I_{u}\left(r_{u}, s_{u}\right), e_{u}, t_{u}$ on the card

Figure 2: Card Initialization

The certificate consists of a pair of integers which is denoted as $\left(r_{s}, s_{s}\right)$ for the server (terminal) and $\left(r_{u}, s_{u}\right)$ for the user (smart card). Here $r_{u}$ and $r_{s}$ are the $x$ coordinates of the (distinct) elliptic curve points $R_{u}$ and $R_{s}$, respectively. As mentioned earlier, the proposed protocol is based on the ECDSA.

\subsection{Mutual Authentication Between Card and Terminal}

This part of the algorithm is used to protect the terminal-card negotiation against eavesdropping especially in contact-less cards where this negotiation takes place over the air. It also ensures the authenticity of each party to the 
other one to avoid using fake cards or fraudulent terminals. The protocols shown in Figures 1 and 2 are executed off-line. The mutual authentication and key agreement protocols between the terminal and the card need to be executed in real-time. We give the combined protocol in Figure 3.

CARD

- Receive

- Send

- $Q_{k}=d_{u} * Q_{s}=\left(d_{u} . d_{s}\right) * P$

- $Q_{k} \cdot x$ : The mutually agreed key

- Receive

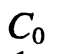

- $D\left(Q_{k} \cdot x, C_{0}\right)$

- $C_{1}=E\left(Q_{k} \cdot x,\left(e_{u},\left(r_{u}, s_{u}\right), t_{u}, g\right)\right)$

- Send

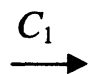

- If $g$ and $t_{u}$ are valid, then

- $c=s_{s}^{-1}$

- $u_{1}=c . e_{s}$

- $u_{2}=c \cdot r_{s}$

- $R=u_{1} * P+u_{2} * Q_{c a}$

- $v=R \cdot x$

- If $v \neq r_{s}$, then abort

- $k_{m}=Q_{k} \cdot x+g$

- $Q_{f}=H\left(k_{m}\right) * Q_{k}$

- $k_{f}=Q_{f} x$ : The unique secret key

\section{TERMINAL OR SERVER}

- Send

- Receive

- $Q_{k}=d_{s} * Q_{u}=\left(d_{s} \cdot d_{u}\right) * P$

- $Q_{k} \cdot x$ : The mutually agreed key

- Generate a random number $g$

- $C_{0}=E\left(Q_{k} \cdot x,\left(e_{s},\left(r_{s}, s_{s}\right), t_{s}, g\right)\right)$

- Send

- Receive

- $D\left(Q_{k} \cdot x, C_{1}\right)$

Figure 3: Mutual Authentication and Key Agreement

According to the protocol, whenever there is a service request either by the card or by the terminal, there is an immediate key exchange. Sending the public keys unprotected over the air (as in contact-less case) does not introduce any threat to the security of the system. Once both sides have the other party's public key, they simultaneously generate a secret key to encrypt the data required to have a mutual authentication. This task is accomplished by multiplying the other party's public key $Q_{2}$ with this party's private key $d_{1}$ as shown in Fig. 3. To protect the certificates from an eavesdropper, it is necessary to send the certificates in encrypted form. For this reason, the 
protocol uses a secret key cipher to encrypt the certificates using the mutually agreed secret key $Q_{k} . x$. The server encrypts the concatenation of its certificate $e_{s},\left(r_{s}, s_{s}\right)$, the certificate expiration date $t_{s}$, and a random number $g$ which will be used to obtain the final mutual key of the communication. The certificates are usually sent in clear in almost all the other authentication protocols. In our protocol, we do not reveal the content of the certificate, which may be useful for spoofing attacks. This increases the encryption time only slightly since the certificate is not very long (on the order kilobits) and the encryption algorithms are very fast (on the order of megabits per second).

The encrypted message $C_{0}$ is then sent to the user. The user then decrypts $C_{0}$ and obtains the certificate of the server and the random number $g$. The user immediately encrypts the concatenation of its certificate $e_{u},\left(r_{u}, s_{u}\right)$, the certificate expiration date $t_{u}$, and the random number $g$. This encrypted data which is denoted as $C_{1}$ is sent to the server. Next, the user checks the validity of the certificate, and if it is invalid, the user aborts the communication. On the other side, the server decrypts $C_{1}$ and checks whether $g$ and the time certificate are valid. If not, it aborts. This mechanism, specifically the use of $g$, defeats spoofing attacks by the user side and also prevents unnecessary computation. Next, the server checks the validity of the certificate and accordingly grants or aborts the service. Note that it may be a good idea to generate multiple $g$ values in advance so that the protocol could save some time since data storage on the terminal side is not a problem. However, storing these multiple random numbers will increase the storage requirement of the protocol, which is undesirable.

After the verification procedure has been completed by both sides, the user and the server are now ready to use the channel that has been reserved for their communication. However, there is one more step to complete our goal to have a full secure protocol: To generate a secret key known by each side to encrypt the conversation. They do already have a secret key $Q_{k} \cdot x$; however, this key cannot be used since it will be the same during the valid time limits of their certificates. Therefore, we need to add a new key exchange step to agree on a unique key to be used for communication during each session. However, we do not prefer to execute another key agreement process due to previously stated power and memory limitations. Instead, we will use the previously generated random number $g$, which is known by both sides, to generate a new number $k_{m}$ without using the channel again. Both the server and the user perform a scalar addition to obtain $k_{m}$. Then $k_{m}$ is hashed, reduced modulo the curve cardinality $n$, and multiplied by the point $Q_{k}$ to give the point $Q_{f}$. The new secret key, which we call $k_{f}$, is taken to be $Q_{f} x$. This key can now be used for encrypting the data sent through the channel. 
This is more secure than simply using $k_{m}$ as the secret key, since $k_{m}$ is related to the first few steps of the protocol.

\section{PERFORMANCE COMPARISON}

ECC delivers the highest strength per bit of any known public-key system because of the difficulty of the hard problem upon which it is based. This greater difficulty of the hard problem - the elliptic curve discrete logarithm problem (ECDLP) - means that smaller key sizes yield equivalent levels of security. Table 1 compare the key sizes needed for equivalent strength security in ECC with RSA and DSA [5]. Given the best known algorithms to factor integers and compute elliptic curve logarithms, the key sizes are considered to be equivalent strength based on MIPS years needed to recover one key.

Table 1: Key Size Equivalent Strength Comparison

\begin{tabular}{||c|c|c|c||}
\hline $\begin{array}{c}\text { Time to } \\
\text { break in } \\
\text { MIPS years }\end{array}$ & $\begin{array}{c}\text { RSA/DSA } \\
\text { key size }\end{array}$ & $\begin{array}{c}\text { ECC } \\
\text { key size }\end{array}$ & $\begin{array}{c}\text { RSA/ECC } \\
\text { key size } \\
\text { ratio }\end{array}$ \\
\hline $10^{4}$ & 512 & 106 & $5: 1$ \\
$10^{8}$ & 768 & 132 & $6: 1$ \\
$10^{11}$ & 1,024 & 160 & $7: 1$ \\
$10^{20}$ & 2,048 & 210 & $10: 1$ \\
$10^{78}$ & 21,000 & 600 & $35: 1$ \\
\hline
\end{tabular}

Table 2 shows the digital signature timings for different public key systems [5]. Here ECDSA shows the best performance characteristics.

Table 2: Digital signature timings (in milliseconds) on a $200 \mathrm{MHz}$ Pentium Pro

\begin{tabular}{||l|c|c|c||}
\hline \multicolumn{1}{|c|}{ Operation } & $\begin{array}{c}\text { RSA- } \\
1024 \\
(e=3)\end{array}$ & DSA-1024 & $\begin{array}{c}\text { ECDSA-168 } \\
(\text { over } G F(p))\end{array}$ \\
\hline Sign & 43 & 7 & 5 \\
Verify & 0.6 & 27 & 19 \\
key generation & 1100 & 7 & 7 \\
parameter generation & none & 6500 & large (research area) \\
\hline
\end{tabular}




\section{IMPLEMENTATION RELATED ISSUES}

The speed of the elliptic curve operations, e.g., the point addition and point multiplication, depends on the arithmetic of the underlying finite field. The drafted IEEE ECC standard proposes the use of the fields $G F(p)$ and $G F\left(2^{k}\right)$. The implementation of the field $G F(p)$ requires that we implement modular arithmetic with respect to a prime modulus $p$. Due to the security requirements, the size of $p$ is at least 100 bits, usually around 160 bits. The large number arithmetic has been extensively studied in the context of the RSA algorithm, and efficient algorithms for field multiplication have been designed. An efficient method for performing the field multiplication is the Montgomery method which effectively performs modulo $2^{k}$ multiplication instead of modulo $p$ multiplication, where $2^{k}>p>2^{k}-1$. Selection of a Mersenne prime (a prime of the form $2^{k}-1$ ) or a prime in the special form of $2^{k}-c$, where $c$ is a small odd integer has also shown to be useful. With the use of these methods, it is possible to obtain high-speed implementations of the elliptic curve cryptographic algorithms.

In general software environments, the use of $G F\left(2^{k}\right)$ offers significant performance advantages over $G F(p)$. This holds true for embedded system, and more importantly, for a low-cost, 8-bit smart card. To achieve a higher performance with $G F(p)$, a crypto coprocessor (a dedicated hardware component for cryptographic processing) is required. This additional crypto coprocessor increases the cost of each chip by 20 to 30 percent, which adds three to five dollars to the cost of the card. With $G F\left(2^{k}\right)$, a smart card is less expensive because a coprocessor is not needed to deliver high performance. In software environments in which an arithmetic processor is already available for modular exponentiation, the performance of $G F(p)$ can be improved so that in some cases it exceeds the performance of $G F\left(2^{k}\right)$. This holds true for platforms such as those using Pentium processors or, in the case of smart cards, those having a crypto coprocessor to accelerate modular arithmetic. If a crypto coprocessor were already available on the smart card (or if the cost associated with adding one were not an issue), then $G F(p)$ would offer performance advantages over $G F\left(2^{k}\right)$ implemented without a dedicated hardware component.

Point compression allows the points on an elliptic curve to be represented with fewer bits of data. In smart card implementations, point compression is essential because it reduces the storage space for keys and certificates on the card in addition to reducing the amount of data that needs to be transmitted to and from the card. 


\section{CONCLUSION}

Smart cards have extremely rigid constraints on processing power, parameter storage, and code space, as well as slow input/output. As a result, implementation of public-key cryptosystems in smart cards has usually been associated with high-end cards, typically with both large memory configurations and a cryptographic coprocessor.

The proposed protocol protects both parties, i.e. the card issuer and the cardholder, against fraud. It uses ECC to enable both sides to communicate securely and efficiently under the constrained storage and processing limitations of the smart card technology. The protocol also provides certain security services, e.g., non-repudiation, anonymity of user, service expiration mechanism using time certificates, as most recent secret and public-key based protocols also provide. The protocol is based on the elliptic curve digital signature algorithm (ECDSA), and inherits the security and implementation properties of the elliptic curve cryptosystems. With a 160-bit modulus, an elliptic curve system seems to offer the same level of cryptographic security as DSA or RSA with 1024-bit moduli.

\section{APPENDIX}

Elliptic curves have been used to factorize numbers and in primality proving as well as cryptography. ECC provides a way to create digital signatures and to construct key exchange protocols. For cryptographic applications the elliptic curves defined over finite fields are of primary interest. The finite fields that are usually used in elliptic curve cryptosystems are the field of prime characteristic $G F(p)$ and the field of characteristic two $G F\left(2^{m}\right)$ [7] [8] [9]. An addition rule is available that can add two points on the curve to obtain a third point on the curve such that:

$P+Q=R$ where $P, Q$ and $R$ are three points on the curve and,

$$
P+O=O+P=P
$$

Multiplication of a point by an integer $n$ is a repeated addition of the point to itself $n$ times: $n * P=P+P+P+\ldots$ ( $n$ times). Addition and multiplication of points are the main operations. They can be implemented in hardware or software and they have efficient mathematical algorithms to compute them. Elliptic curve multiplication is analogous to exponentiation in discrete logarithm cryptosystems, i.e., it is an operation that is easy to compute but very difficult and time consuming to reverse. 


\section{- Curves in fields of characteristic $p$ :}

In this case the curve equation is $y^{2}=x^{3}+a x+b$ and the addition rule is defined as follows:

$P_{3}\left(x_{3}, y_{3}\right)=P_{1}\left(x_{1}, y_{1}\right)+P_{2}\left(x_{2}, y_{2}\right), P_{3} \neq O$

where $x_{3}=\lambda^{2}-x_{1}-x_{2}$,

$y_{3}=\left(x_{1}-x_{3}\right) \lambda-y_{1}$

and $\lambda=\left(y_{2}-y_{1}\right) /\left(x_{2}-x_{1}\right)$ when $x_{1} \neq x_{2}$

or $\lambda=\left(3 x_{1}^{2}+a\right) / 2 y_{1}$ when $x_{1}=x_{2}, y_{1} \neq 0$

\section{- Curves in fields of characteristic 2:}

In this case the curve equation is $y^{2}+x y=x^{3}+a x^{2}+b$ and the addition rule is defined as follows:

$P_{3}\left(x_{3}, y_{3}\right)=P_{1}\left(x_{1}, y_{1}\right)+P_{2}\left(x_{2}, y_{2}\right), P_{3} \neq O$

where $x_{3}=\lambda^{2}+\lambda+a+x_{1}+x_{2}$,

$y_{3}=\left(x_{1}+x_{3}\right) \lambda+x_{3}+y_{1}$

and $\lambda=\left(y_{2}+y_{1}\right) /\left(x_{2}+x_{1}\right)$ when $x_{1} \neq x_{2}$

or $\lambda=\left(x_{1}^{2}+y_{1}\right) / x_{1}$ when $x_{1}=x_{2} \neq 0$

\section{REFERENCES}

[1] W. Diffie and M. Hellman, "New directions in cryptography", IEEE Trans. Inform. Theory, vol. IT-30, 1976.

[2] T. ElGamal, "A public key cryptosystem and a signature scheme based on discrete logarithms", IEEE Trans. Inform. Theory, vol. IT-31, 1985.

[3] S. Pohlig and M. Hellman, "An improved algorithm for computing logarithms over GF(p) and its cryptographic significance", IEEE Trans. Inform. Theory, vol. IT-24, 1978.

[4] D. Chaum, A. Fiat and M. Naor, "Untraceable electronic cash", Advances in Cryptology, Crypto 88, S. Goldwasser (Ed.), SpringerVerlag.

[5] Michael J. Wiener, "Performance Comparison of Public-Key Cryptosystems", CryptoBytes, vol.4, number 1- summer 1998

[6] M. Aydos, B. Sunar, C. K. Koc, "An Elliptic Curve Cryptography based Authentication and Key Agreement Protocol for Wireless Communication", $2^{\text {nd }}$ International Workshop on Discrete Algorithms and Methods for Mobile Computing, 1998

[7] Henry Beker and Fred Piper, "Cipher Systems", Northwood Publications, 1982.

[8] William Stallings, "Cryptography and Network Security: Principles and Practice", Second Edition, Prentice Hall, 1999.

[9] Alfred J. Menezes, Paul C. van Oorschot and Scott A. Vanstone, "Handbook of Applied Cryptography",CRC Press, 1997. 